\title{
Clinical Outcomes from Tamoxifen Drug-herb Interactions
}

\section{Campos Maria Graça ${ }^{1,2^{\star}}$, Mariana Cupido ${ }^{1}$, Raquel Tavares $^{1}$ and Raquel Consul $^{1}$}

${ }^{1}$ Observatory of Drug-Herb Interactions/ Faculty of Pharmacy, University of Coimbra, Health Sciences Campus, Azinhaga de Santa Comba, Coimbra, Portugal

${ }^{2}$ Coimbra Chemistry Centre, University of Coimbra, Rua Larga, Coimbra, Portugal

\section{Abstract}

\section{Publication History:}

Background: Tamoxifen is an important drug in chemotherapy being part of various protocols in cancer treatment, but is not universally effective even when used before surgery or in preventing recurrence. Pharmacogenetic variability in drug metabolism is one possible mechanism of treatment failure. We hypothesize that Drug-Herb Interactions (DHI) contribute in disease outcomes, nevertheless the functional single nucleotide polymorphisms in drug metabolizing enzymes that activate cytochrome enzymes.

Methods: We performed a retrospective study in the last 9 years of clinical cases enrolled in follow up of Observatory of Drug-Herb Interactions, University of Coimbra - Portugal (www.oipm.uc.pt), and the data collected will be discussed in this paper. Data obtained from PubMed and from PubChem Compounds with preference given to the data obtained during the last 10 years, was also obtained. The search terms were varied depending of the Clinical situation.

Results: From our experience, in order to avoid the major predictable DHI more observational trials should be carried out for therapeutic protocols associated to tamoxifen. The most consumed, fruits and vegetables, medicinal plants and other natural products associated to the intake of tamoxifen were, for example, Orange and Better juice, Aloe, Geranium, Saint John Wort leaves and flowers, roots as Astragalus, Curcuma, Ginger, Ginseng, Rehmanniae and Valeriana, mushrooms as Coriolus, Maitake, Shiitake and Reishi.

Conclusions: Based on the major compounds involved in those products will be predictable how to advice patients doing this kind of treatment and follow a possible therapy failure, or even a toxic event. Our group did a flyer with the main possible interactions selected for DHI with Tamoxifen. This it will be given to patients doing therapy with tamoxifen, helping them to be a aware of the situation and involving all in the resolution for a better outcome (it will be available in our website as other materials for download already posted there).

\section{Introduction}

Tamoxifen can be used in several ways, as for instance, to lower the chances of cancer coming back and is usually taken for 5 to 10 years but can be prescribed before surgery too (neoadjuvant therapy) [1]. In both situations, the effectiveness of the drug is crucial for the outcome. However, the result sometimes is unexpected.

Two major points for the therapeutic ineffectiveness are pointed out, one related to genetic polymorphisms and the other include Drug-Herb Interactions (DHI). Firstly, the genetic idiosyncrasy, since that $7 \%$ of the Caucasian population are slowly (poor metabolizers) of CYP2D6 [2]. Goetz et al. [3] published some years ago that "in tamoxifen-treated breast cancer patients, women with a variant allele of cytochrome $P-4502 D 6$ enzyme, $C Y P 2 D 6^{*} 4{ }^{*} 4$ genotype, have a higher risk of disease relapse demonstrated by relapse-free time and diseasefree survival". In the same year, these data was corroborate by Knox et al, showing that "the 2-year relapse-free survival was $68 \%$ in patients who are poor metabolizers of CYP2D6 and patients on strong inhibitors of CYP2D6 compared to 98\% in patients who are extensive metabolizers of CYP2D6" [4]. Secondly, Tamoxifen is one among other drugs that suffer often DHI with one of the two possible outcomes, increased side effects or therapeutic failure.

Until 1990 these DHI were mainly with food and, in their clinical trials for various drugs, before the approval, this situation was studied. However, in this decade the legislation of "Dietetic supplements" has changed in the United States of America, and later in Europe. With this change, many medicinal plants were included in these products and patients are harassed to consume them. Basically, in the previous
Received: May 31, 2018

Accepted: August 06, 2018

Published: August 08, 2018

\section{Keywords:}

Drug-Herb interactions, Clinical outcomes, Breast cancer, Inhibitors, Dietetic supplements definition of "Dietetic supplements", they contain "proteins, minerals and vitamins", to that content was added "herbs and similar products", and then was allowed to industry to add medicinal plants [5].

Many of the DHI that we will discuss in the results became from these products.

Food supplements aim to maintain physiological homeostasis, medicinal products aim to prevent or treat diseases [6] but, as it is pointed out by the Bureau Européen des Unions des Consommateurs Aisbl, unfortunately the use of "both nutrients and botanicals in food supplements may pose risks for human health once for minerals or vitamins the risk lies with potential overdose while not all botanicals are safe for use in food supplement"... "In particular, the term 'botanical' may have several confusing meanings and/or synonyms. The same botanical may be used simultaneously in a food supplement and as a medicinal product, depending on the product's intended use. However, both food supplements and medicinal products may be available in the same form i.e. herbs, powders, pills or tablets. All these complexities

"Corresponding Author: Dr. Campos Maria Graça, Observatory of DrugHerb Interactions/ Faculty of Pharmacy, University of Coimbra, Health Sciences Campus, Azinhaga de Santa Comba, Coimbra Portugal; E-mail: mgcampos@ff.uc.pt

Citation: Graça CM, Cupido M, Tavares R, Consul R (2018) Clinical Outcomes from Tamoxifen Drug-herb Interactions. Int J Clin Pharmacol Pharmacother 3: 140. doi: https://doi.org/10.15344/2456-3501/2018/140

Copyright: (C) 2018 Graça et al. This is an open-access article distributed under the terms of the Creative Commons Attribution License, which permits unrestricted use, distribution, and reproduction in any medium, provided the original author and source are credited. 
Citation: Graça CM, Cupido M, Tavares R, Consul R (2018) Clinical Outcomes from Tamoxifen Drug-herb Interactions. Int J Clin Pharmacol Pharmacother 3: 140. doi: https://doi.org/10.15344/2456-3501/2018/140

Page 2 of 6

make it difficult for consumers to distinguish between the thousands of products labelled as 'herbal', 'botanical', "natural supplement", 'plant food supplement herbal medicinal product" or even "medical device" [7].

To further complicate the situation the main target of these products is mainly focus in Chronic Diseases patients and Oncology, began a complex process of DHI.

Nonetheless, the supposed benefit is not clear the damage suffers a lack of proper care and gave rise to a series of accidents caused by the indiscriminate use of "natural products" with bioactivity/toxicity. Since oncological patients became easy targets for the sale of these products, the intake in self-medication with conventional therapies, as Tamoxifen, is unpredictable.

We study various potential DHI, based in the experience of OIPM/ FFUC follow-up of oncologic patients. Our purpose is to bring the issue for an open discussion and give tools to the total involvement of health professionals and patients in its treatments to prevent backto-hospital adverse effects, with toxic activity outside the limits of the revised for this type of treatments, or even treatment failures.

\section{Methods}

We performed a retrospective study in the last 9 years of clinical cases enrolled in follow up of Observatory of Drug-Herb Interactions, University of Coimbra (OIPM/FFUC) - Portugal (www.oipm.uc.pt), and the data collect will be discussed in this paper.

The study was observational and the results given are an evidence based medicine approach. Information from the literature was carried out on different databases such as DrugBank ${ }^{\circ}$, Medscape ${ }^{\circ}$, PubMed $^{\circ}$ and Medline ${ }^{\oplus}$ were consulted between March 2009 and April 2018 using the search terms "tamoxifen", "CYP", "juice", "herbs", "herbdrug" and "interactions", as so, the specific terms of each particular clinical case, in order to identify citations, abstracts and articles. Some more specific information about each plant was also collected from databases such as Natural Medicines Comprehensive Database, National Centre for Complementary and Alternative Medicine and Medscape Reference.

Ethical and Clinical permission regarding the study was approved in May 2009 included in the project "IciPlant - Interactions between cytostatic drugs and Plants" developed at the Coimbra Oncologic Hospital and coordinated by OIPM/FFUC (2009-2020). The data provided belong to preliminary approach to the issue and for that reason there is no statistical data to provide.

\section{Results and Discussion}

Oncologic patients are aware that its chemotherapy is one of the most aggressive and are afraid of side effects. Sometimes they take "natural products" as self-medication, or even recommended by different "players" as internet sales promotions, herbalists and/or complementary therapies. Briefly, the supposed end-point is to reduce side effects doing a detoxification and increase the immune system. "Natural products" involved in drug discovery research are also the subject of sales without being careful to assess that, in most cases, there have been no product safety tests yet, and the toxicity of the product puts patients at risk.

Int J Clin Pharmacol Pharmacother

ISSN: 2456-3501
Patients are not aware of these issues or even of the possible DHI that could occur with this concomitant intake and what they should do to maintain the plasma concentration of the drug in the right levels, decreasing the possibility of this undesirable symptoms or even therapeutic failures.

For a better understanding of DHI, in the text below, the presentation of the results is followed of the discussion and will be divided in three points. Firstly, the mechanisms of potential interactions with the main estrogen-like natural products commonly used in concomitance with Tamoxifen; secondly, the risks associated with the use of inhibitors and/or inducers of CYP isoenzymes and carrying proteins; to finish some examples of angiogenic potential plants to be avoided. At the end, summarize data collected from the clinical reports is included in Table 1, for an easy overview.

\section{Potential interactions of main estrogen-like natural products}

Tamoxifen is an antagonist of the action of estrogen in the breast and used, among other oncologic indications, in treatment of estrogen receptor positive breast cancer, early and advanced, in pre- and postmenopausal women and in men. It can be prescribed for prevention of breast cancer in women that are at high risk for breast cancer. This drug acts as a weak estrogen in bone tissue but as a strong antagonist of the action of estrogen in the breast, being called a selective estrogen receptor modulator (SERM) and some DHI could occur at this level [8].

Estrogen or antiestrogen bioactivity of some natural products should be avoided to prevent a potential DHI. A precaution must be taken when Caucasian patients ingest for example, isoflavones [9] from Astragalus membranaceus (Fisch.) (astragalus), Cimicifuga ramosa L. (black cohosh)[10], Glycine soja L. (soy) [11], or even from (-)-secoisolariciresinol, a lignin present in Linum usitatissimum L. (flax seeds) [12] and Glycyrrhiza glabra (Licorice root). Various medicinal plants from China are estrogen-like as, for instance, roots of Rehmannia glutinosa L., Paeoniae alba, Angelicae sinensis L. [13]. These compounds have estrogen-like activity and act as endocrine disruptors inducing cell proliferation what is a risk for cancer patients. Data from Asians is different, once polymorphisms produce different metabolites [14].

\section{Risks associated with the use of inhibitors and/or inducers of CYP isoenzymes and carrying proteins}

Tamoxifen is extensively converted to active metabolites in humans by hepatic cytochrome P450 enzymes, being CYP3A4 and CYP2D6 the most relevant in the metabolic process. These metabolites may be responsible for many of the effects of the tamoxifen. Therefore, DHI with Tamoxifen are very important to assure that the main biologically active metabolite is produced in the right plasma concentration, and to prevent a fast elimination too. Since the main active metabolite is made by the action of CYP2D6, drugs that are powerful inhibitors of the enzyme's activity may reduce the effects of Tamoxifen and thus lead to therapeutic ineffectiveness $[2,8]$. As CYP3A4 contributes to the bioactivation of tamoxifen, drugs that inhibit the activity will lead to a decrease in the formation of the active metabolites (in both possible metabolic pathways), with consequent therapeutic ineffectiveness $[2,15]$.

From the main concerns in Tamoxifen Summary of Product Characteristics (SmPC) we emphasize "In the literature it has been shown that CYP2D6 poor metabolizers have a lowered plasma level of 
Citation: Graça CM, Cupido M, Tavares R, Consul R (2018) Clinical Outcomes from Tamoxifen Drug-herb Interactions. Int J Clin Pharmacol Pharmacother 3: 140. doi: https://doi.org/10.15344/2456-3501/2018/140

Page 3 of 6

endoxifen, one of the most important active metabolites of tamoxifen. Concomitant medications that inhibit CYP 2D6 may lead to reduced concentrations of the active metabolite endoxifen. Therefore, potent inhibitors of CYP 2D6 (e.g. paroxetine, fluoxetine, quinidine, cinacalcet or bupropion) should whenever possible be avoided during tamoxifen treatment" and it also advertising that "When Tamoxifen Tablets are used in combination with coumarin-type anticoagulants, a significant increase in anticoagulant effect may occur"... and as "tamoxifen is metabolised by cytochrome P450 3A4, care is required when coadministering with drugs, such as rifampicin, known to induce this enzyme as tamoxifen levels may be reduced. The clinical relevance of this reduction is unknown" [16].

As various Medicinal Plants, some sold as "botanicals" in Food Supplements, can interfere with CYP3A4 and CYP2D6 and cause $\mathrm{DHI}$, but from the literature is not easy getting them directly with tamoxifen. The relevance of the data collected from other drugs and from clinical reports with our patients, will help to highlight some events and contribute in a near future to better to better adjust the treatment, avoiding unpredictable outcomes.

In some countries as our, patients are often harassed to take Aloe vera (L.) Burm. f. prepared in different ways but mainly as syrup made as an alcoholic beverage. The extract include anthraquinones and some were study as cytotoxic but they are also potent laxatives causing various side effects in the patients, including lesions in intestine, that could be favorable to opportunistic infections once chemotherapy has immunosuppressive effect [17]. A few studies were carried out with this cactus and even in a study designed to evaluate in vitro inhibition of these enzymes for ethanolic extract of Aloe vera latex (Aloe barbadensis Miller), it was concluded that there was inhibition of CYP3A4 and CYP2D6 by a double mechanism of inhibition (reversibly and irreversibly), with a decrease in the active metabolites and, thus, therapeutic ineffectiveness [18].

Other potential inhibitors of CYP3A4 are extracts from roots of Curcuma, Ginger, Ginseng and Valerian [19]. It should be adjusted the dose of tamoxifen if the patient insist in the intake, otherwise the bioactivation of Tamoxifen will be compromise.

Caffeic acid and/or its derivatives present in many medicinal plants as Camellia sinensis L. (green and black tea, among others), Cimicifuga racemosa L. (black cohosh), Geranium robertianum L. (herb Robert) [19], Matricaria recutita L. (matricaria), Melissa officinalis L. (balm), Rosmarinus officinalis (rosemary), Salvia officinalis L. (salvia), Verbena officinalis L. (verbena), among others, as in coffee, olive oil,

\begin{tabular}{|c|c|c|c|}
\hline $\begin{array}{l}\text { Natural Product/ bioactive } \\
\text { compounds }\end{array}$ & Estrogenic activity & Angiogenic bioactivity & CYP interference \\
\hline $\begin{array}{l}\text { Aloe/antraquinones and } \\
\beta \text {-sitosterol }\end{array}$ & No data & $\begin{array}{l}\text { Angiogenic/ } \\
\text { Antiangiogenic }[23,24]\end{array}$ & $\begin{array}{l}\text { Inhibitor CYP 3A4, Inhibitor CYP } 2 \mathrm{~A} 6 \nrightarrow \text {, } \\
*[18]\end{array}$ \\
\hline Astragalus /isoflavones & $\begin{array}{l}\text { Estrogen-like activity in Caucasians/ } \\
\text { antiestrogenic for Asians [14] }\end{array}$ & Angiogenic [24] & No data \\
\hline Angelica & & Angiogenic [24] & \\
\hline $\begin{array}{l}\text { Black cohosh/isoflavones } \\
\text { and caffeic derivative acids }\end{array}$ & $\begin{array}{l}\text { Estrogen-like activity in Caucasians } \\
\text { Antiestrogenic for Asians }[10,14]\end{array}$ & & $\begin{array}{l}\text { Small inhibitory effect on CYP2D6;possible } \\
\text { inhibitory effect on CYP3A4 [25] }\end{array}$ \\
\hline Coriolus versicolor & No data & Antiangiogenic [24] & Inhibitor CYP $3 \mathrm{~A} 4 \nrightarrow[26]$ \\
\hline Curcuma & No data & Anti-angiogenic $\nrightarrow[27]$ & $\begin{array}{l}\text { Inhibits CYP 1A2, 3A4, CYP 2C9 and 2D6 } \\
\leftrightarrow \text { [27], Induces CYP 2A6 [28] }\end{array}$ \\
\hline Echinacea & No data & & Inhibits CYP3A4 $[22,29]$ \\
\hline Geranium & No data & & Inhibits CYP 3A4 [19] \\
\hline Ginger & No data & $\begin{array}{l}\text { Anti-angiogenic } \\
{[24], \text { dose-dependent }} \\
{[30,31]}\end{array}$ & Inhibits CYP 3A4 $\nrightarrow[30]$ \\
\hline Panax ginseng & $\begin{array}{l}\text { No data(hormonal activity does not } \\
\text { involve estrogen receptors) }\end{array}$ & Angiogenic [24] & Inhibits CYP 3A4 and CYP2D6 $\nrightarrow[32]$ \\
\hline Green tea & No data & Anti-angiogenic $[33]$ & $\begin{array}{l}\text { inhibited P-gp-mediated efflux activity, } \\
\text { inhibited CYP2C9, 2D6, and 3A4 } \leftrightarrow[\text { [34] }\end{array}$ \\
\hline Licorice root & Hormonal activity & Anti-angiogenic $[24,35]$ & $\begin{array}{l}\text { Inhibits CYP3A4 } \nrightarrow[36] \text { Weak Inhibitor of } \\
\text { CYP2D6 } \nrightarrow[36]\end{array}$ \\
\hline Reichi & No data & Anti-angiogenic [24] & Inhibits CYP 3A4 [37] \\
\hline Rehmannia & & Angiogenic [21] & \\
\hline Rosemary & No data & & \\
\hline Saint John Worth & No data & & Induces CYP $3 \mathrm{~A} 44^{*}[38]$ \\
\hline Salvia miltiorrhiza & & Angiogenic [24] & \\
\hline Soy/isoflavones & $\begin{array}{l}\text { Estrogen-like activity in Caucasians } \\
\text { Antiestrogenic for Asians }[11,14]\end{array}$ & & Inhibits 1A2, CYP3A4 [14] \\
\hline Valeriana & & & Inhibits CYP3A4 and CYP2D6 $\nrightarrow[39]$ \\
\hline Verbena & & & Inhibits CYP 3A4 $\nrightarrow[21]$ \\
\hline
\end{tabular}

Table 1: Major Drug-Herb Interactions found for Tamoxifen in Portugal. 
Citation: Graça CM, Cupido M, Tavares R, Consul R (2018) Clinical Outcomes from Tamoxifen Drug-herb Interactions. Int J Clin Pharmacol Pharmacother 3: 140. doi: https://doi.org/10.15344/2456-3501/2018/140

Page 4 of 6

many fruits and vegetables, wine, bee pollen and propolis, making them potential inhibitors of $2 \mathrm{C} 9$ and CYP3A4 [20]. From the medicinal plants cited above, in our clinical reports we had DHI with Aloe vera, Geranium and Verbena, that are in line with this hypothesis, reinforcing the potential risk and alerts should be given by the health professionals to the patients about these possible situations. The fact that we do not have notifications of the DHI with other plants cited above does not mean that they cannot occur.

Since the wide variety of plants and food products containing caffeic acid and its derivatives, as well as other compounds, it is important to recommend to the patient for do not change their diet during treatment, because the Tamoxifen dose is adjusted according to the usual diet. Any change in it, as introduction of supplementation, increased coffee intake or infusions, among others) should be reported to the doctor, before taking them, so that the risk can be assessed.

Orange Juice and St. John's Worth, can increase the rate of elimination of drugs by CYP3A4 and P-glycoprotein induction but in the case of Tamoxifen, depending of the extent of the interaction, sometimes the increase of the secondary metabolites could arise to a toxic event. Despite that, in our patients taking high levels of orange juice (3 oranges/ two times a day) as possible detox product the therapeutic failure occurs in concomitance with diarrhea too.

Some of our patients also take medicinal mushrooms. They are mostly used in Asia and nowadays commercialized in Europe for different purposes even for cancer treatments associated to Conventional therapies. Some of the more commonly used species by our patients include Coriolus versicolor (turkey tail), Ganoderma lucidum (reishi), Grifola frondosa (maitake), Lentinus edodes (shiitake), and Poriacocos (Schw.) Wolf. The main raison, as they say, is because in herbal stores, internet and by some Complementary therapists, they are encouraged to take those products to stimulate immune system. Despite a possible beneficial effect, not fully established, DHI should be evaluated and patients follow-up include possible changes in absorption, due to polysaccharides content of mushrooms and CYP interactions although they have not yet been duly clarified.

Considering the cases that have arisen during these 9 years in the OIPM/FFUC and taking into account that clinical outcomes were collected with Caucasian patients, we could infer that the therapeutic ineffectiveness was not due to the genetic polymorphism of CYP2D6, as could be implicit from the discussion did above. Since, by eliminating the inhibitory and / or inducing agents of the enzyme complex in question, the efficacy of tamoxifen therapy has been restored to have plasma concentration values within the range of therapeutic window.

Another issue that is discussed sometimes but in the majority of the threatments is not taken in account, is that Tamoxifen and metabolites endoxifen and 4-hydroxy-tamoxifen exposure could be associated to seasonal variations, with considerable higher plasma concentrations during summer. Recently data concluded, what is already known, that CYP3A4 contributes to the bioactivation of Tamoxifen through formation of 4-hydroxy-tamoxifen and becomes increasingly important in case of reduced or absent CYP2D6 activity. Seasonal variations also influence the plasma concentration levels of the active metabolites, being higher in the summer (there is greater exposure to vitamin D) [21]. It is known that there are nuclear receptors responsible for the induction of metabolizing enzymes, among which we can highlight Pregnane X receptor (PRX) and Vitamin-D-binding receptor (VDR). PXR is the steroid and xenobiotic receptor and VDR is the vitamin D receptor. These receptors, when active, will lead to a response at the level of genetic transcription since this mechanism aims to detoxify and eliminate the xenobiotic. PXR likewise regulates the expression of CYP3A4 as well as VDR. During the summer, with increased sun exposure, there is more production of vitamin $\mathrm{D}$ and this, when it binds to VDR, will induce CYP3A4 (among other enzymes) [22]. In countries as our, with high levels of sun exposure, particular care could be taken since an increase of the metabolization of tamoxifen ultimately increase the concentrations of active metabolites at this time of year.

\section{Angiogenic potential plants}

Angiogenesis is the growth of neovessels from existing vasculature and angiogenic diseases result from new blood vessels growing either excessively, as in cancer, diabetic retinopathy and psoriasis. It is consider a key step in tumor growth, invasion and metastasis [23].

Is not easy to make a statement that help to prevent these potential interactions. The selection of several plants is very difficult to do, since sometimes for the same product they appear with angiogenic and anti-angiogenic activity in literature, as for instance, Aloe vera, Panax ginseng, Vitis spp. (grape). Anyway, from those controversial results, a special information should be given to patients in order to prevent a greater vascularization of the tumor process; in the meantime, we wait for more robust assays associated with angiogenic activity.

Others as Astragalus membranaceus, Angelica sinensis, Rehmannia glutinosa (Jun) and Salvia miltiorrhiza are potential angiogenic plants and the risk associated to the intake is more clear [23,24].

All of them, were unfortunately recommended to some of our patients as co-adjuvants of the treatments, by the players cited above, and the outcomes involved DHI in various clinical reports in addition to a potential vascularization of the tumor in others. As an example occurred in one of our clinical reports involving the intake of Panax ginseng in a Caucasian patient, 58 years old, with a meningioma in the sellar region medicated with Tamoxifen. The drug was ineffective because Ginseng inhibits CYP3A4 and CYP2D6 and also contributes to the increase angiogenesis. Briefly, in the first surgery, an increased vascularization was verified and the procedure had to be suspended. Following the suspension of the plant, a decrease in vascularization was observed in the following surgical process, as well as a recession of the meningioma, which allowed a safe removal.

\section{Conclusions}

From our experience, in order to avoid the major predictable DrugHerb Interactions with Tamoxifen more "Observational controlled studies", should be carried out to be sure of the real impact in oncologic population by ethnic group. The global dissemination of research data allow sales of "natural products", by people that do not follow the legislation in what concerns to the rules of efficacy, safety and quality and put patients in risk.

The most consumed products by our patients are, for example, Astragalus, Angelica, Orange and Better juice, Aloe, Curcuma, Saint John Wort, Ginger, Ginseng, Rehmannia, Salvia and mushrooms as Coriolus, Maitake, Shiitake and Reishi. 
Citation: Graça CM, Cupido M, Tavares R, Consul R (2018) Clinical Outcomes from Tamoxifen Drug-herb Interactions. Int J Clin Pharmacol Pharmacother 3: 140. doi: https://doi.org/10.15344/2456-3501/2018/140

Page 5 of 6

Health professionals should be updated in this Drug-Herb Interactions for better help patients to take the right decisions. Among them, Pharmacists and Pharmaceutical care is a very important "tool" to avoid this type of situation since it lets a closer monitoring of the patient, which allows a better education that leads to safer choices. It is also important to understand why patients are looking for these alternative substances and realize that some events are occurring with conventional therapy because patients usually fear adverse effects. It is important then to explain how medications work, to alert to these issues and thus decrease the risk of therapeutic failure.

All these outcomes could give consensus around specific items necessary for evaluating risk failure making Tamoxifen more effective.

\section{Author's contributions}

Maria Graça Campos: Coordinator of the project and involved in the resolution of the clinical events. Information collected from databases and Discussion of the data. Contribute with the writing of the manuscript.

Mariana Cupido: Involved in the resolution of the clinical events. Information collected from databases and Discussion of the data. Contribute with the writing of the manuscript.

Raquel Tavares: Involved in the resolution of the clinical events. Information collected from databases and Discussion of the data Contribute with the writing of the manuscript.

Raquel Consul: Involved in the resolution of the clinical events. Information collected from databases and Discussion of the data. Contribute with the writing of the manuscript.

\section{Competing Interests}

The authors declares that they have no competing interests.

\section{References}

1. Hormone Therapy for Breast Cancer.

2. Krämer SD, Testa B (2008) The Biochemistry of Drug Metabolism - An Introduction. Part 6. Inter-Individual Factors Affecting Drug Metabolism. Chem Biodivers 5: 2465-2578.

3. Goetz MP, Rae JM, Suman VJ, Safgren SL, Ames MM, et al. (2005) Pharmacogenetics of tamoxifen biotransformation is associated with clinical outcomes of efficacy and hot flashes. J Clin Oncol 23: 9312-9318.

4. Knox SK, Ingle JN, Suman VJ, Rae JM, Flockhart DA, et al. (2006) Cytochrome P450 2D6 status predicts breast cancer relapse in women receiving adjuvant tamoxifen. J Clin Oncol 24: 504.

5. Worsnop RL (1993) Dietary Supplements: Should health products be less tightly regulated?

6. Commission staff working document (2008) Available scientific information on the use of substances other than vitamins and minerals in food supplements. Council of Europe.

7. Passaranillaria (2016) Food Supplements. Challenges \& risks for consumers Bureau EuropéenDes Unions De Consommateurs Aisbl. Der Europäische Verbraucher verband.

8. Consortium on Breast Cancer Pharmacogenomics (2008) A Guide for Breast Cancer Patients and Physicians. Drug-Interactions With Tamoxifen.

9. Leclercq G, Jacquot $Y$ (2012) Interactions of isoflavones and other plant derived estrogens with estrogen receptors for prevention and treatment of breast cancer-Considerations concerning related efficacy and safety. Steroid Biochem Mol Biol 139: 237-244.
10. Silva $A G$, Brandão $A B$, Cacciari RS, Soares WH (2009) Avanços na elucidação dos mecanismos de ação de Cimicifugaracemosa (L.) Nutt. Nos sintomas do climatério. Rev Bras Plantas Med 11: 455-464.

11. Jargin SV (2014) Soy and phytoestrogens: possible side effects. Ger Med Sci 12: 18.

12. Ghazanfarpour $M$, Sadeghi R, Latifnejad $R$, Khadivzadeh $T$, Khorsand $I_{\text {, }}$ et al. (2016) Effects of flaxseed and Hypericumperforatum on hot flash, vaginal atrophy and estrogen-dependent cancers in menopausal women: A systematic review and meta-analysis. Avicenna J Phytomed 6: 273-283.

13. Hao Q, Wang J, Niu J, Zhao P, Cui Y, et al. (2009) Study on phytoestrogeniclike effects of four kinds of Chinese medicine including Radix Rehmanniae Preparata, Radix Paeoniae Alba, Radix Angelicae Sinensis, Rhizoma Chuanxiong. Zhongguo Zhong Yao Za Zhi 34: 620-624.

14. Campos MG, Costa ML (2012) Possible Risks in Caucasians by Consumption of Isoflavones Extracts Based. In Structure and Function of Food Engineering 9: 205-224.

15. Antunes MV, Timm TAF, Olivera V, Staudt DE, Gossling G, et al (2015) Influence of CYP2D6 and CYP3A4 phenotypes, drug interactions and vitamin D status on tamoxifen biotransformation. Ther Drug Monit 37: 733 744.

16. Tamoxifen Summary of Product Characteristics.

17. Jesus NR, Batista C, Silva CM, Silva TP, Costa ML, et al. (2016) Oncological Patients Under Therapy and Potential Risks Associated to Interactions with Natural Products for Constipation Relieve. Int J Clin Pharmacol Pharmacother 1: 117.

18. Djuv A, Nilsen OG (2012) Aloe vera juice: IC $_{50}$ and dual mechanistic inhibition of CYP3A4 and CYP2D6. Phytother Res 26:445-451.

19. Sun DX, Lu JC, Fang ZZ, Zhang YY, Cao YF, et al (2010) Reversible inhibition of three important human liver cytochrome p450 enzymes by tiliroside. Phytother Res 24: 1670-1675.

20. Jaikang C, Niwatananun K, Narongchai P, Narongchai S, Chaiyasut C, et al. (2011) Inhibitory effect of caffeic acid and its derivatives on human live cytochrome P450 3A4 activity. Journal of Medicinal Plants Research 5:35303536.

21. Antunes MV, Timm TAF, Olivera V, Staudt DE, Gossling G, et al. (2015) Influence of CYP2D6 and CYP3A4 phenotypes, drug interactions and vitamin D status on tamoxifen biotransformation. Ther Drug Monit 37: 733744.

22. Meijerman I, Beijnem JH, Schellens JH (2006) Herb-Drug Interaction in Oncology: Focus on Mechanisms of Induction. Oncologist 11: 742-752.

23. Sahib HB, Harchan NAH, AtraqchiSAM, Abbas AA (2010) The Role of Medicinal Herbs in Angiogenesis Related Diseases. International Journal of Pharmacology 6: 616-623.

24. Tai-Ping F, Yeh JC, Leungc KW, Yued PYK, Wongcd NS (2006) Angiogenesis: from plants to blood vessels. Trends Pharmacol Sci 27: 297-309.

25. Gurley BJ, Gardner SF, Hubbard MA, Williams DK, Gentry WB, et al. (2005) In vivo effects of goldenseal, kava kava, black cohosh, and valerian on human cytochrome P450 1A2, 2D6, 2E1, and 3A4/5 phenotypes. Clin Pharmacol Ther 77: 415-426.

26. Yeung JH, Or PM (2012) Polysaccharide peptides from Coriolus versicolor competitively inhibit model cytochrome P450 enzyme probe substrates metabolism in human liver microsomes. Phytomedicine 19: 457- 463.

27. Bamba Y, Yun YS, Kunugi A, Inoue H (2011) Compounds isolated from Curcuma aromatic Salisb. inhibit human P450 enzymes. J Nat Med 65: 583587.

28. Chen Y, Liu WH, Chen BL, Fan L, Wang G, et al (2010) Plant Polyphenol Curcumin Significantly Affects CYP1A2 and CYP2A6 Activity in Healthy, Male Chinese Volunteers. Ann Pharmacother 44: 1038-1045.

29. Scott GN, Elmer GW (2002) Update on natural product-drug interactions. Am J Health-Syst Pharm 59: 339-347.

30. Hyeong JK, In SK, Shaheed UR, Sang KH, Katsunori N, et al. (2017) Effects of 6-paradol, an unsaturated ketone from gingers, on cytochrome P450mediated drug metabolism. Bioorg Med Chem Lett 27: 1826-1830.

31. Park JH, Park GM, Kim JK (2015) Zerumbone, Sesquiterpene Photochemical from Ginger, Inhibits Angiogenesis. Korean J Physiol Pharmacol 19: 335340.

32. Wanwimolruk S, Phopin K, Prachayasittikul V (2014) Cytochrome P450 enzyme mediated herbal drug interactions (Part 2). EXCLI J 13: 869-896. 
Citation: Graça CM, Cupido M, Tavares R, Consul R (2018) Clinical Outcomes from Tamoxifen Drug-herb Interactions. Int J Clin Pharmacol Pharmacother 3: 140. doi: https://doi.org/10.15344/2456-3501/2018/140

Page 6 of 6

33. Sagar S, Yance D, Wong R (2006) Natural health products that inhibit angiogenesis: a potential source for investigational new agents to treat cancer-Part 2. Curr Oncol 13: 99-107.

34. Nishikawa M, Ariyoshi N, Kotani A, Ishii I, Nakamura H, et al. (2004) Effects of continuous ingestion of green tea or grape seed extracts on the pharmacokinetics of midazolam. Drug Metab Pharmacokinet 19: 280-289.

35. Sagar S, Yance D, Wong R (2006) Natural health products that inhibit angiogenesis: a potential source for investigational new agents to treat cancer-Part 2. Curr Oncol 13: 99-107.

36. He W, Wu JJ, Ning J, Hou J, Xin $\mathrm{H}$, et al. (2015) Inhibition of human cytochrome P450 enzymes by licochalcone A, a naturally occurring constituent of licorice. Toxicol In Vitro 29: 1569-1576.

37. Chen S, Yong T, Zhang Y, Su J, Jiao CY, et al. (2017) Anti-tumor and Antiangiogenic Ergosterols from Ganodermalucidum. Front Chem 5: 85.

38. Goey AK, Meijerman I, Rosing H, Marchetti S, Mergui-Roelvink M, et al. (2014) The Effect of St John's Wort on the Pharmacokinetics of Docetaxel. Clin Pharmacokinet 76: 467-474.

39. Nowack R (2008) Review Article: Cytochrome P450 enzyme, and transport protein mediated herb-drug interactions in renal transplant patients: Grapefruit juice, St John's Wort and beyond. Nephrology 13: 337-347. 